\title{
Thermal and hygroscopic expansion characteristics of bamboo
}

Puxi Huang BEng, MSc, PhD

Researcher, Department of Architecture and Civil Engineering,

University of Bath, Bath, UK (corresponding author: P.Huang@bath.ac.uk) (Orcid:0000-0003-4512-6072)

Wen-shao Chang BS Arch, MS Arch, PhD, FHEA

Senior lecturer, School of Architecture, University of Sheffield, Sheffield, UK (Orcid:0000-0002-2218-001X)

Martin P. Ansell BSC, PhD, FIMMM

Reader, Department of Architecture and Civil Engineering, University of Bath, Bath, UK (Orcid:0000-0003-2946-1735)
Chris Rhys Bowen BSc, PhD

Professor, Department of Mechanical Engineering, University of Bath, Bath, UK (Orcid:0000-0002-5880-9131)

John Y. M. Chew BCom, BEng, PhD (Cantab), CEng, CSci,

MIChemE

Senior lecturer, Department of Chemical Engineering, University of Bath, Bath, UK (Orcid:0000-0003-2888-834X)

Vana Adamaki BSc, MSc, PhD

Research associate, Department of Mechanical Engineering, University of Bath, Bath, UK

The expansion and contraction of bamboo caused by temperature and moisture variations must be evaluated if bamboo is to be utilised as a building material. However, detailed expansion data, especially data in the ascent and descent processes of temperature and moisture are unexplored. The aim of this study is to investigate the expansion characteristics of Phyllostachys edulis (Moso bamboo) in ascent and descent processes of temperature and moisture. The measurement of linear thermal expansion and hygroexpansion of bamboo specimens were conducted with a dilatometer and a micrometer, respectively. The temperature range of the linear thermal expansion measurement was between 23 and $70^{\circ} \mathrm{C}$. In the linear moisture expansion measurement, all specimens were measured at various relative humidity $(\mathrm{RH})$ conditions including oven dry, 27, 54, 76 and $95 \% \mathrm{RH}$, and water soaking stages. Major findings include: the swell of bamboo specimens increases with $\mathrm{RH}$, whereas the shrinkage of bamboo specimens decreases with $\mathrm{RH}$. The hygroexpansion is much more significant compared with the linear thermal expansion. Both linear thermal expansion and hygroexpansion of the specimens from the longitudinal directions are smaller than those from the radial direction and tangential direction.

\section{Notation}

$l \quad$ length of the specimen in equilibrium state $(\mathrm{mm})$

$l_{0} \quad$ length of the specimen in oven-dry state $(\mathrm{mm})$

$S_{\mathrm{e}} \quad$ expansion rate $(\%)$

$\delta l / l \quad$ expansion value (\%)

\section{Introduction}

This study investigated the linear thermal and hygroexpansion characteristics of Phyllostachys edulis (Moso bamboo) in three directions with respect to the cylindrical coordinate system. As a biological building material resource, bamboo can be potentially implemented as components of building envelopes owing to its fast reproductive capacity and competitive thermal and mechanical properties (Flander and Rovers, 2009; Majumdar et al., 2010; Van Der Lugt et al., 2006). To assess the structural stability of bamboo in different climatic conditions, expansion and contraction data are indispensable when variations in temperature and moisture occur. Linearly, the expansion and contraction are similar to the Cauchy strain, which can be expressed by the ratio of the increased or decreased length to the initial length (Cverna, 2002). Temperature and humidity are two common driving potentials of the expansion or contraction for bamboo. Thermal expansion is utilised to describe the deformation by temperature variation, while the hygroexpansion, namely shrinking and swelling, is used for deformation caused by relative humidity $(\mathrm{RH})$.
Thermal expansion, shrinking and swelling data of bamboo are still scarce in the literature when compared with a related research on wood. Thermal and moisture strain data of a bamboo plywood wall were measured under periodic temperature and relatively humidity conditions by Li et al. (2012). The effect of utilising bamboo and hybrid bamboo-precipitated calcium carbonate filler to improve the thermal expansion performance of filled plastic composites was assessed by Huang et al. (2012). The linear thermal expansion thermal coefficients of nine wood species and resin-bonded birch laminates were investigated in the three directions of the cylindrical coordinate system (Weatherwax and Stamm, 1956). The hygroexpansion of three wood species under sinusoidally varying RH conditions was studied by Chomcharn and Skaar (1983). The hygroscopic swelling and shrinkage of latewood cell wall was investigated by a stereological technology (Rafsanjani et al., 2014). The hygroexpansion of Cunninghamia lanceolata (Chinese fir) during moisture adsorption and desorption processes was reported by Ma et al. (2005).

In civil engineering, considerable attention needs to be paid to deformation. Otherwise, cracks, undesired strain and movement will lead to serious damage to the appearance and structural properties of buildings. To avoid these problems, it is necessary to understand the value of the expansion or contraction of building materials. This study obtained the expansion features of bamboo specimens in three directions of cylindrical 
coordinates. The results of this study provide criteria for evaluating the deformation and structural stability of Moso bamboo-based building materials.

\section{Methodology}

\subsection{Specimens preparation}

Figures 1(a)-1(c) explain the cutting positions and directions of the bamboo specimens. Three cube specimens and three cuboid specimens were, respectively, cut at both internode and node from the external surface to the internal surface of a Moso bamboo culm. The side length of cube specimens is $3 \pm 0.5 \mathrm{~mm}$. The side length of cuboid specimens is equal to cube specimens in the radial and tangential directions. The side length of cuboid specimens in the longitudinal direction is $23 \pm 0.5 \mathrm{~mm}$. The cube specimens were utilised for thermal expansion measurements and the cuboid specimens were utilised for hygroexpansion measurements. Two different sizes of specimens were used for the following reasons. After a number of initial measurements, the results indicated that the hygroexpansion of bamboo specimens in the longitudinal direction is in a relatively small range. The micrometer has insufficient accuracy to measure longitudinal direction hygroexpansion. Likewise, due to the relatively higher length of the cuboid specimens in the longitudinal direction, the thermal expansion measurements in the radial and tangential directions of cuboid specimens cannot be conducted in the chamber of the dilatometer. To assess the thermal expansion in the radial and tangential directions, the size of cube specimens is more suitable for thermal expansion measurements. The moisture content of all bamboo specimens was reduced to zero in an oven at $103 \pm 2{ }^{\circ} \mathrm{C}$. Then, the specimens were stored in a desiccator with calcium chloride $\left(\mathrm{CaCl}_{2}\right)$ as desiccant.

\subsection{Thermal expansion measurements}

The thermal expansion measurements of the bamboo specimens were conducted with a Netzsch DIL 402C dilatometer. The temperature settings for the linear thermal expansion measurement were from room temperature to $23^{\circ} \mathrm{C}$, from 23 to $70^{\circ} \mathrm{C}$, maintained at $70^{\circ} \mathrm{C}$ for $30 \mathrm{~min}$ and then from 70 to $23^{\circ} \mathrm{C}$. The rate of temperature change was $2^{\circ} \mathrm{C} / \mathrm{min}$. The thermal expansion of the cube specimens was measured in the radial, tangential and longitudinal directions.

\subsection{Moisture expansion measurements}

The hygroexpansion measurements were conducted in desiccators with saturated solutions. The temperature of the experiment was $23 \pm 2^{\circ} \mathrm{C}$. The saturated solutions provide a steady $\mathrm{RH}$ in each desiccator. Four saturated solutions were utilised to determine the $\mathrm{RH}$ conditions in desiccators. Dettol fungicide was added in the potassium nitrate $\left(\mathrm{KNO}_{3}\right)$ solution to avoid undesired mould and mildew growing on the bamboo specimens. The real $\mathrm{RH}$ conditions were also monitored by RH sensors; see Figure 2 and Table 1.
After oven drying, all bamboo specimens were first put into a desiccator with magnesium chloride hexahydrate $\left(\mathrm{MgCl}_{2} \cdot 6 \mathrm{H}_{2} \mathrm{O}\right)$ saturated solution at. Then, these specimens were weighed every $24 \mathrm{~h}$ until the change of length among three consecutive measurements was less than $0 \cdot 1 \%$. The hygroexpansion of each specimen was calculated at this equilibrium state by Equation 1.

$$
\text { 1. } S_{\mathrm{e}}=\frac{l-l_{0}}{l_{0}}
$$

After recording the hygroexpansion, all specimens were moved into a new desiccator. In sorption processes, the moving sequence of desiccators is from oven dry to magnesium chloride hexahydrate to sodium bromide $(\mathrm{NaBr})$ to sodium chloride $(\mathrm{NaCl})$ to potassium nitrate. In the desorption processes, the moving sequence of desiccators is from potassium nitrate to sodium chloride to sodium bromide to magnesium chloride hexahydrate to oven dry.

\section{Results and discussion}

\subsection{Thermal expansion results and discussion}

The thermal expansion results of Moso bamboo specimens are illustrated in Figures 3-8. The left vertical axis is the linear thermal expansion. The right vertical axis represents the temperature of the dilatometer chamber. The horizontal axis shows the experiment time. The temperature setting of the dilatometer is marked by a solid line. The thermal expansion of the external specimen, middle specimen and internal specimen were designated as no. 1 , no. 2 and no. 3 , respectively. The data marker of the no. 1 , no. 2 and no. 3 are solid squares with a solid line, crosses with a solid line and triangles with a solid line, respectively. The actual temperatures of the three specimens in the dilatometer are marked by squares, crosses and triangles, respectively.

The thermal expansion results indicated that all specimens demonstrated an ascending trend in the temperature ascent process. When the temperature was held at $70^{\circ} \mathrm{C}$, contraction occurred in all specimens. During the temperature descent process, a further contraction could be observed in the results. The contraction was mitigated when the temperature returned back to room temperature. Previous studies on thermogravimetric and differential scanning calorimetry analysis reported that a noticeable weight loss, peak value of specific heat capacity and thermal conductivity can be found around $70^{\circ} \mathrm{C}$ (Li et al., 2011; Sun et al., 2006; Wu et al., 2004). This contraction has been attributed to the dehydration of specimens by many researchers (e.g. Dong and Xiong, 2014; Li et al., 2011; Sun et al., 2006). In the temperature ascent process, the thermal expansion values from high to low are external specimens, middle specimens and internal specimens. The density of these specimens increased from the internal side to the external side of the bamboo culm wall; previous thermal 


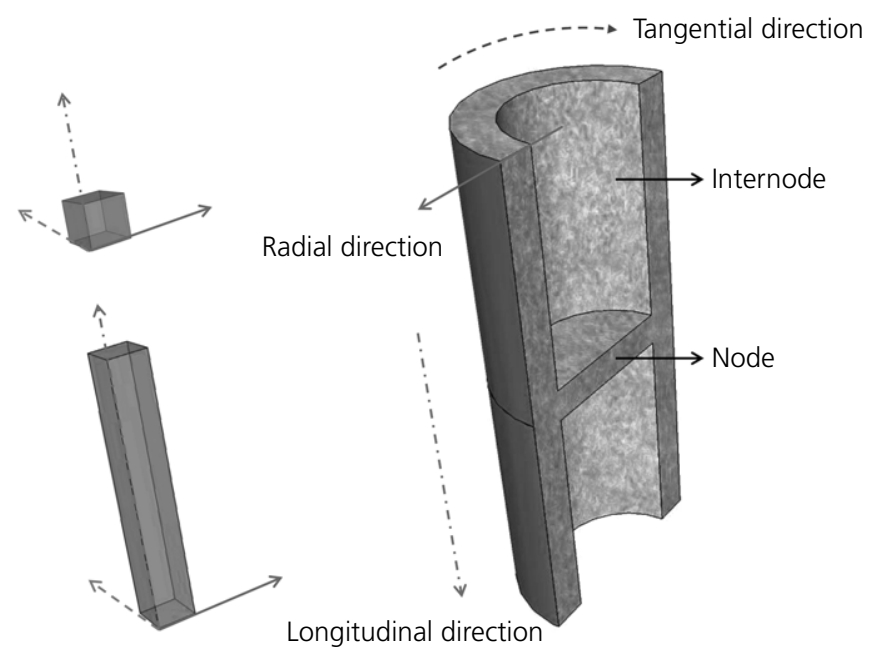

(a)
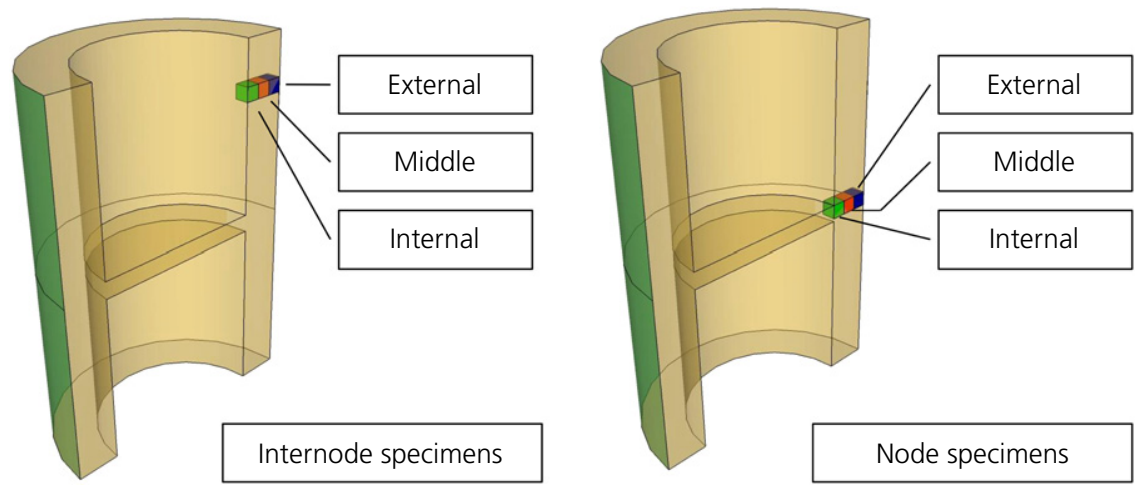

(b)
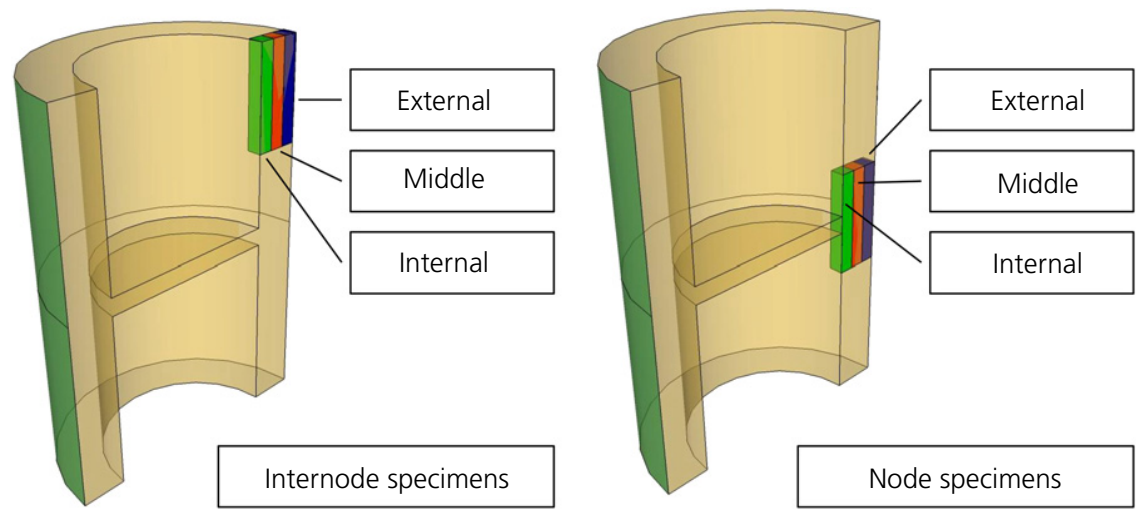

(c)

Figure 1. (a) Cutting position and directions of bamboo specimens. (b) Cutting position of cube bamboo specimens. (c) Cutting position of cuboid bamboo specimens

expansion research on nine wood species indicated that the density can be considered as a factor which influences the thermal expansion (Weatherwax and Stamm, 1956). The thermal expansion of the bamboo specimen in the radial and the tangential direction is one order of magnitude higher than the thermal expansion in the longitudinal direction.
This phenomenon has been reported in many previous papers on wood (Hendershot, 1924; Pizzo et al., 2002; Stevens, 1960). One reason is that the bond energy of fibre tissue is higher along the longitudinal direction than the radial and the tangential directions (Pizzo et al., 2002; Tu and $\mathrm{Xu}, 2008$ ). Hori and Wada (2005) concluded that the crystal structure 
of the wood cellulose dominated the anisotropic thermal expansion in the three coordinate directions and the macroscopic thermal behaviour of solid wood.

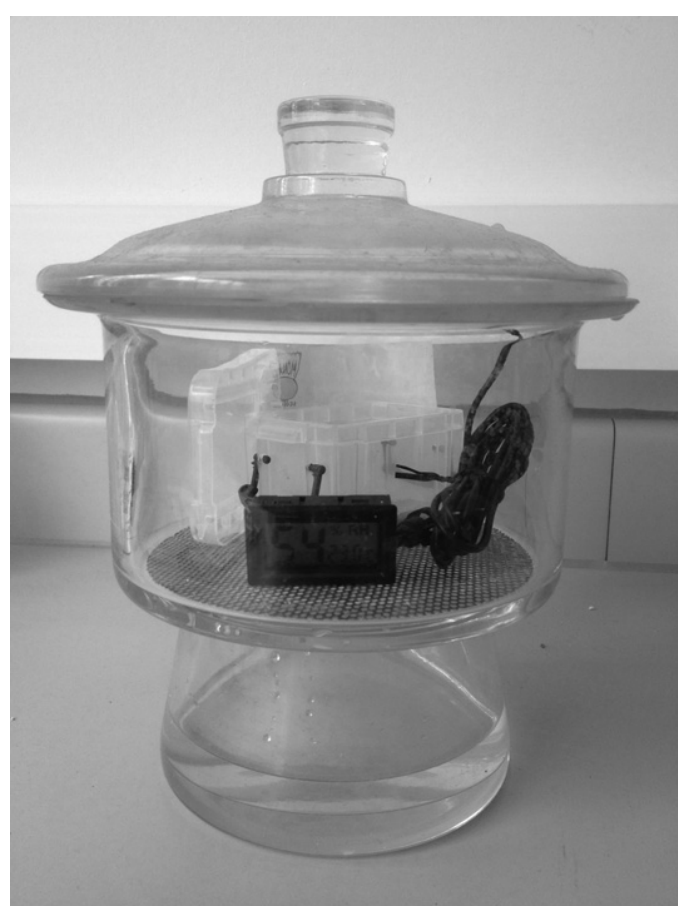

Figure 2. Desiccator assemblies

Table 1. Relative humidities in desiccators

\begin{tabular}{lcc} 
Substance & $\begin{array}{c}\text { Theoretical } \\
\text { RH: \% }\end{array}$ & $\begin{array}{c}\text { Real } \\
\text { RH: \% }\end{array}$ \\
\hline $\begin{array}{l}\text { Magnesium chloride hexahydrate } \\
\left(\mathrm{MgCl} \cdot 6 \mathrm{H}_{2} \mathrm{O}\right)\end{array}$ & 33 & 27 \\
Sodium bromide $(\mathrm{NaBr})$ & 57 & 54 \\
Sodium chloride $(\mathrm{NaCl})$ & 75 & 76 \\
Potassium nitrate $\left(\mathrm{KNO}_{3}\right)$ & 93 & 95
\end{tabular}

\subsection{Hygroexpansion results and discussion}

The hygroexpansion results of Moso bamboo specimens are illustrated by Figures 9-14. The vertical axis represents the hygroexpansion value. The horizontal axis shows the humidity condition of the desiccators. The squares, crosses and triangles represent the external, middle and internal specimens, respectively. The data markers with solid lines are the data for the sorption process. The data markers with dashed lines are the data for the desorption process.

The hygroexpansion results illustrated that the swelling of bamboo specimens increases with the $\mathrm{RH}$ whereas the shrinkage of bamboo specimens decreased with the RH. In the radial direction, the data indicated that the hygroexpansion values from high to low were the external specimen, the middle specimen and the internal specimen. The hygroexpansion in the longitudinal direction is one order of magnitude lower than hygroexpansion in the radial and tangential directions. Tu and $\mathrm{Xu}$ (2008) concluded that the high proportion of vascular bundle tissue and vessels could be regarded as the reason for the high hygroexpansion.

The gross wood structure, the fibre alignment and the layered cell-wall construction have been considered as the factors which influence the hygroexpansion in the radial and tangential directions (Pentoney, 1953). The microfibril angle (MFA) theory was utilised to explain the anisotropic hygroexpansion of wood specimens (Barber and Meylan, 1964). However, previous studies on the MFA of Moso bamboo indicated that the MFA variation of Moso bamboo specimens in the radial and tangential directions was much smaller than the MFA variation of wood ( $\mathrm{Tu}$ and $\mathrm{Xu}, 2008$ ). The MFA cannot be regarded as a decisive factor that influences the hygroexpansion of bamboo (Jiang et al., 2000; Yu et al., 2007) in these directions. However, the structure of the microfibrils is such that water can enlarge the distance between microfibrils but cannot enlarge the length of the microfibrils (Tu and $\mathrm{Xu}, 2008$ ).

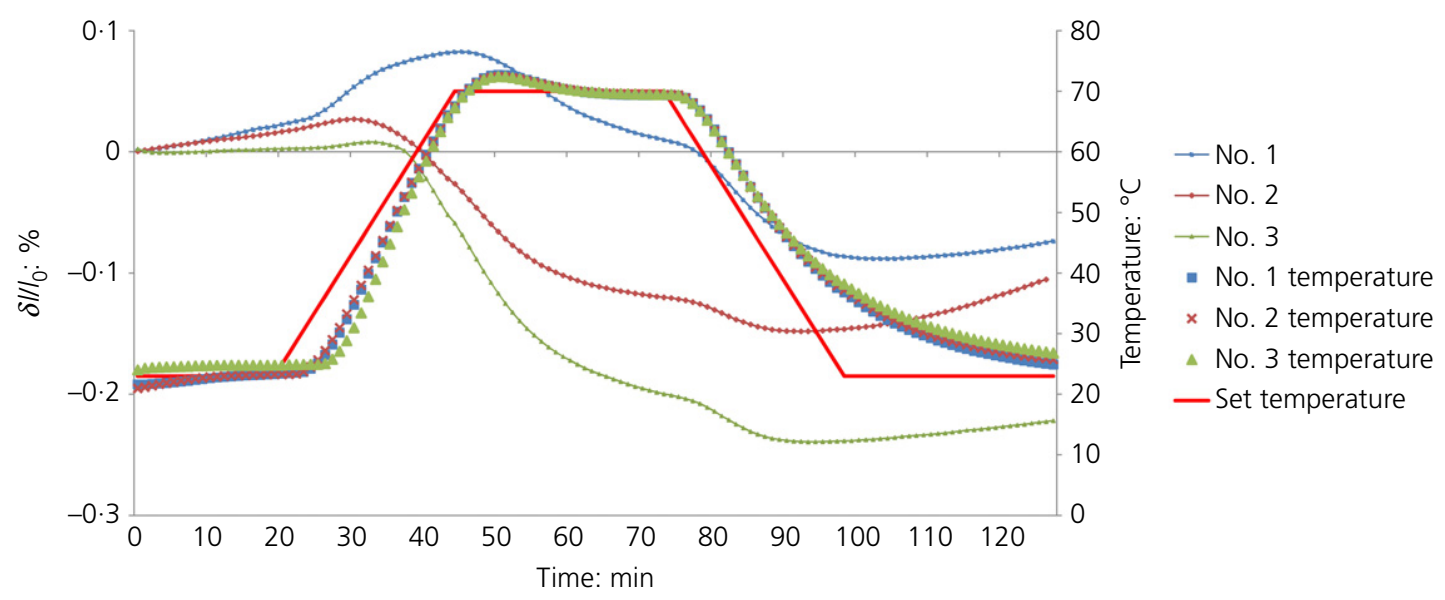

Figure 3. Thermal expansion of internode specimens in the radial direction 


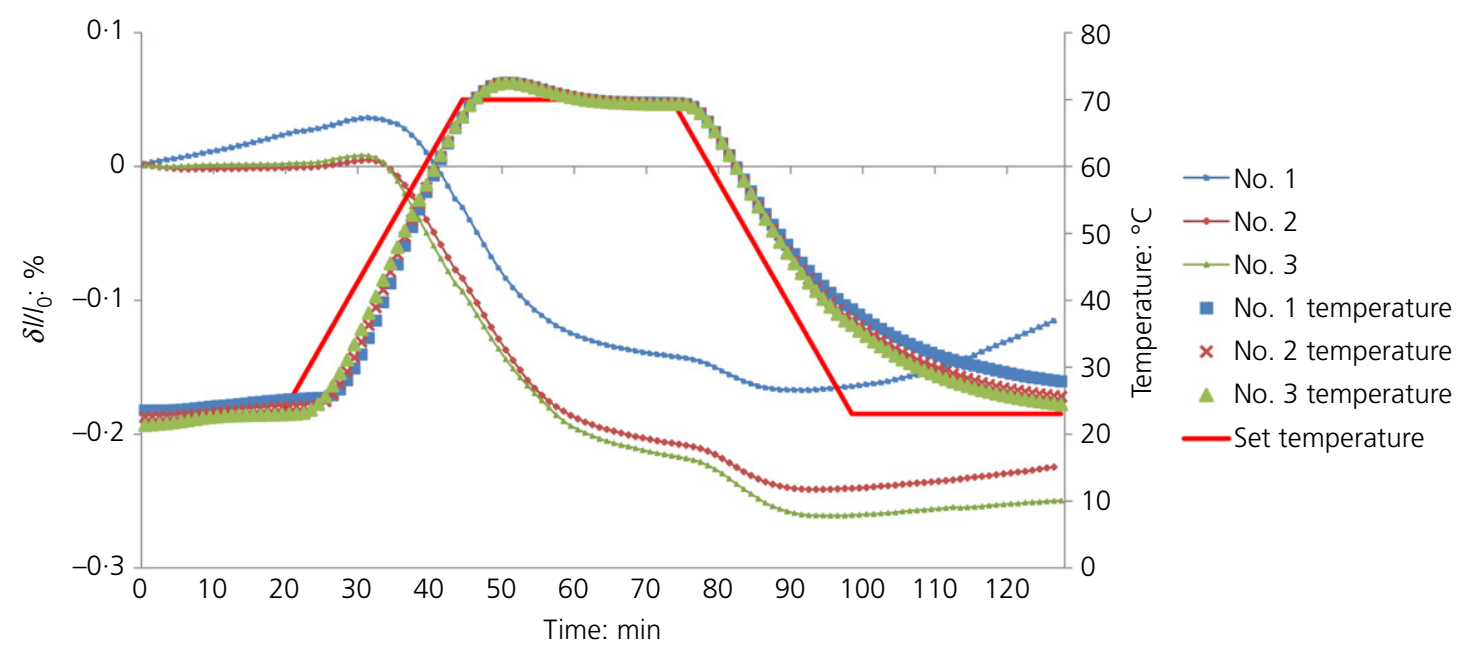

Figure 4. Thermal expansion of node specimens in the radial direction

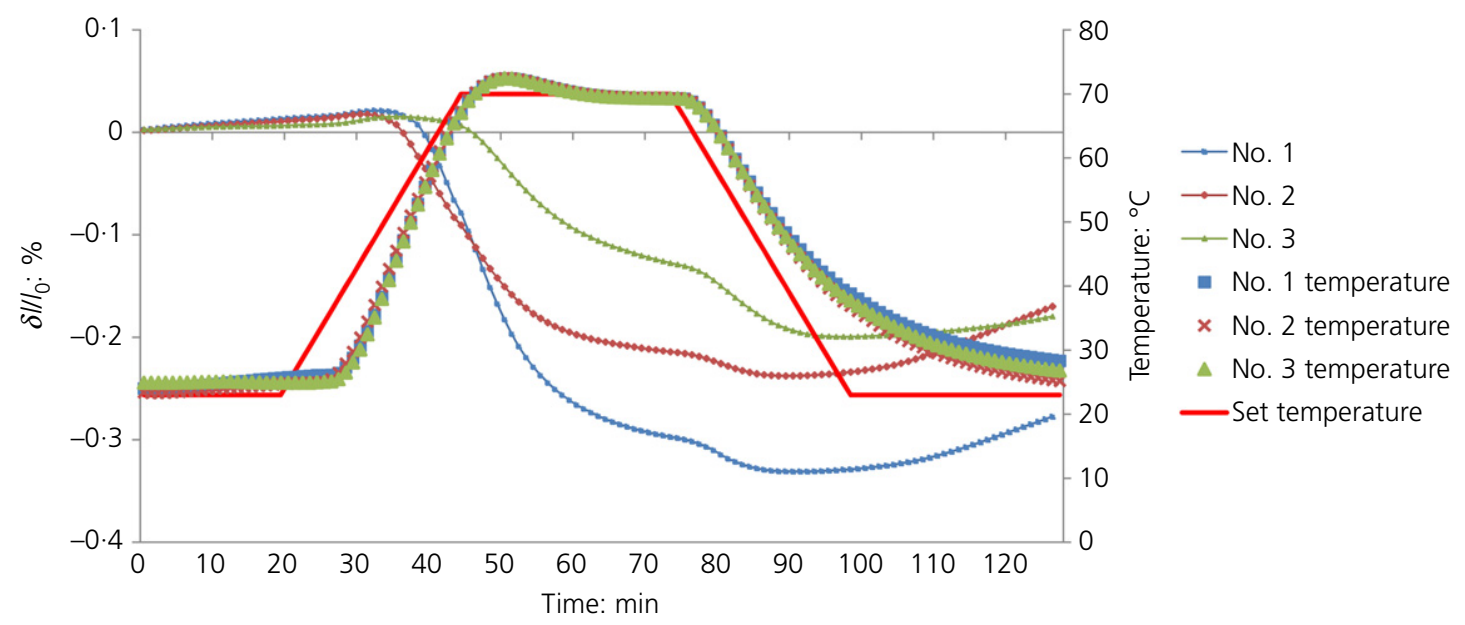

Figure 5. Thermal expansion of internode specimens in the tangential direction

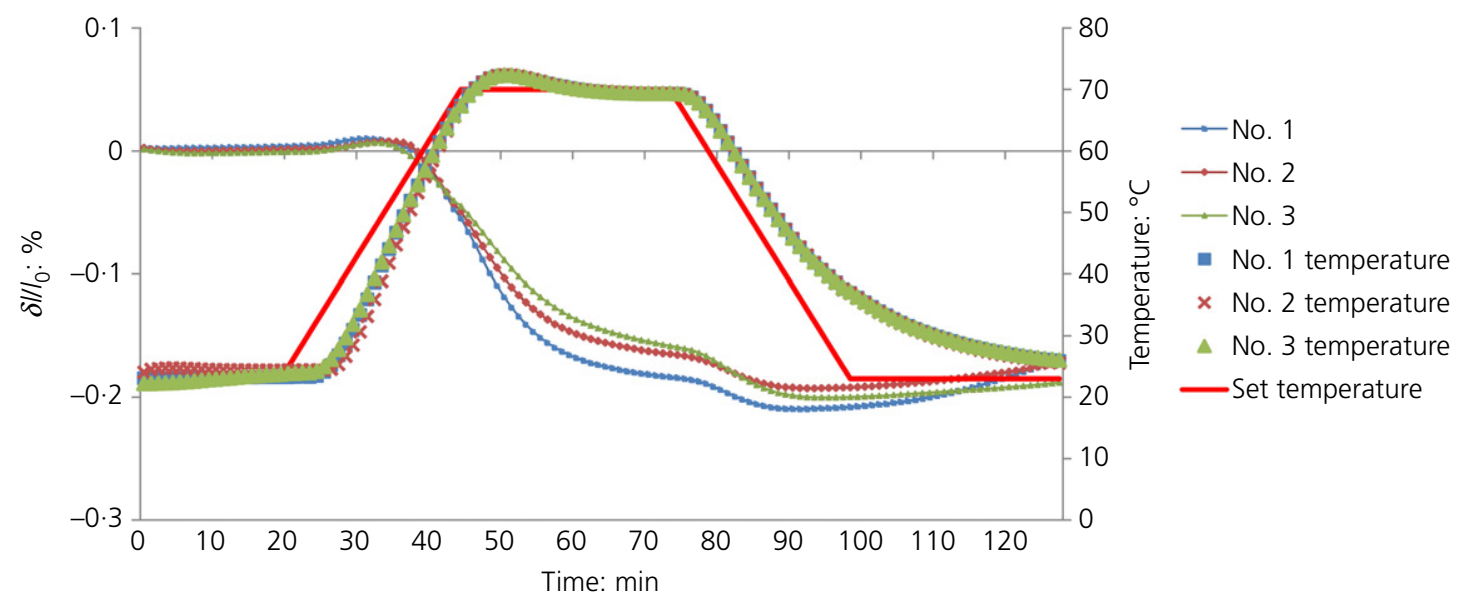

Figure 6. Thermal expansion of node specimens in the tangential direction 


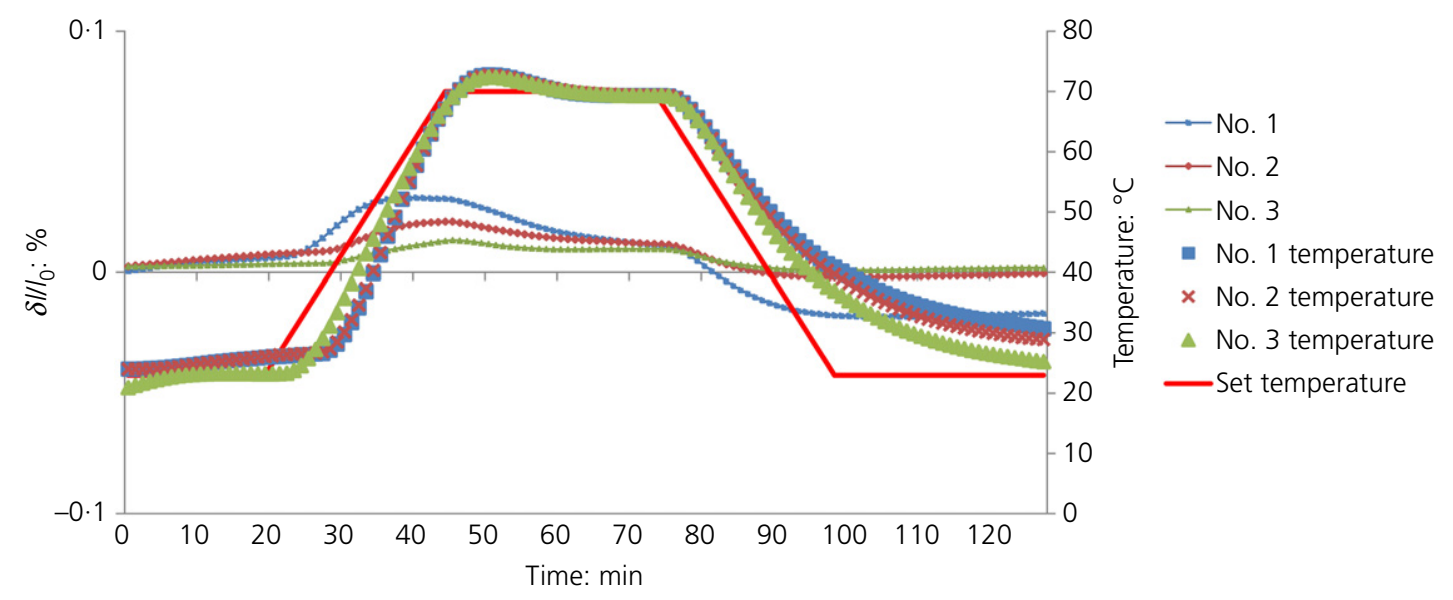

Figure 7. Thermal expansion of internode specimens in the longitudinal direction

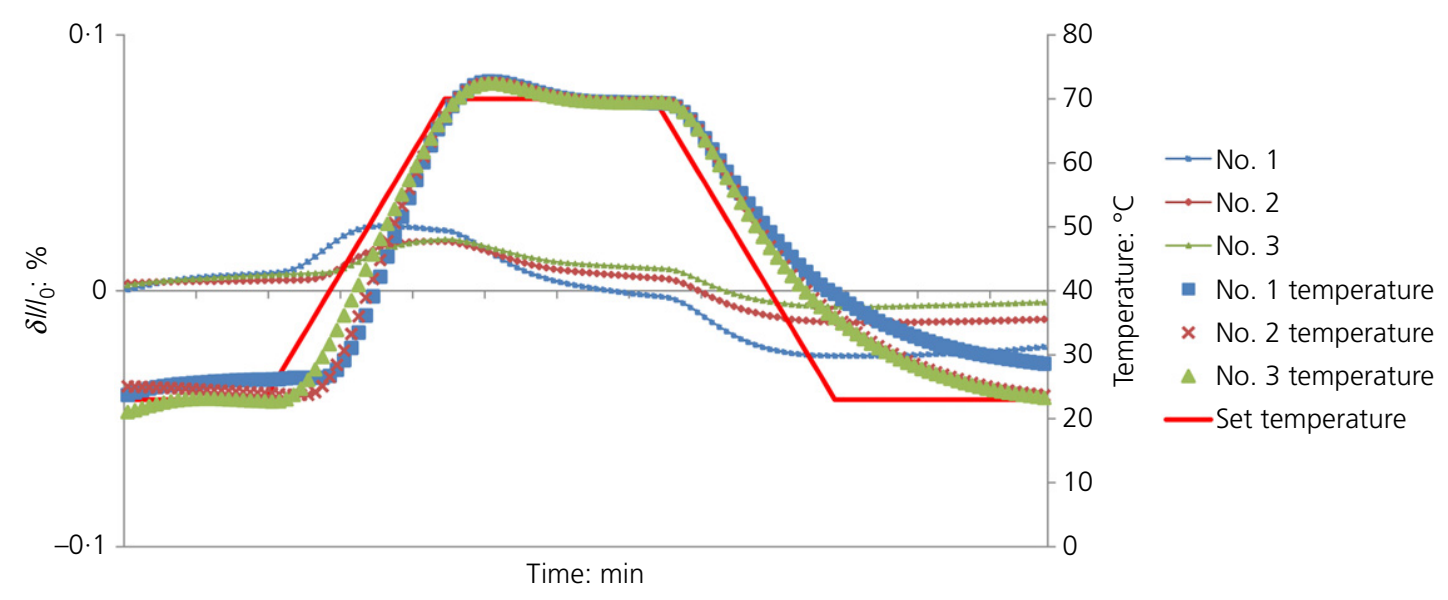

Figure 8. Thermal expansion of node specimens in the longitudinal direction

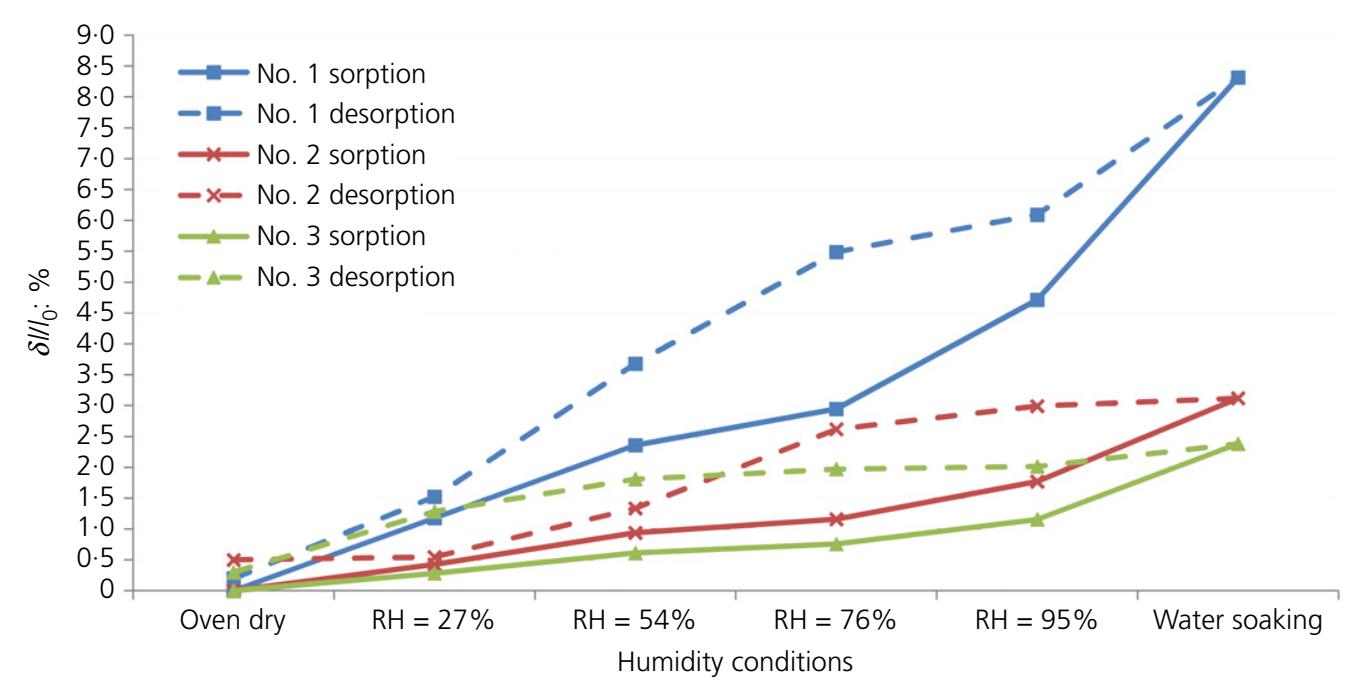

Figure 9. Hygroexpansion of internode specimens in the radial direction 


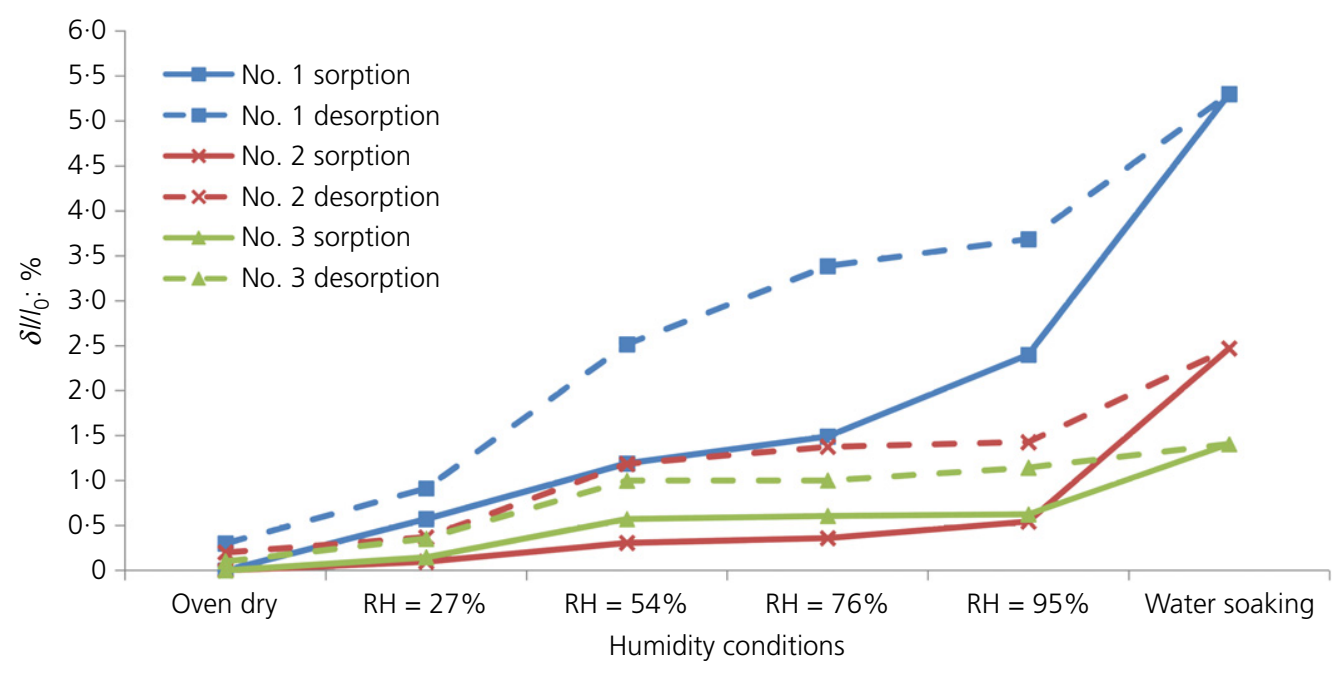

Figure 10. Hygroexpansion of node specimens in the radial direction

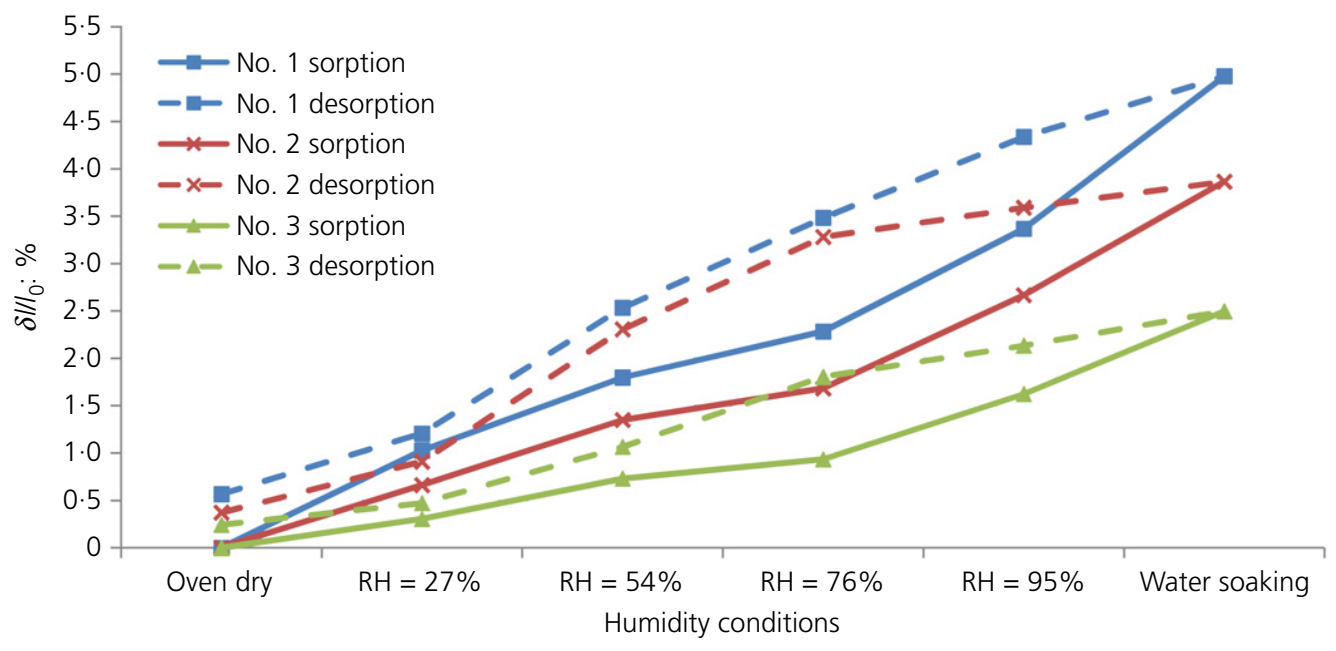

Figure 11. Hygroexpansion of internode specimens in the tangential direction

Research on late wood and early wood indicated that the hygroexpansion of the early wood is highly dominated by the late wood (Pentoney 1953; Skaar, 1988). Although no growth ring can be found in the anatomic structure of bamboo, a remarkable density difference between the vascular bundle tissue and parenchyma ground tissue is similar to the density difference between the early wood and late wood.

The thermal expansion and hygroexpansion results showed that the swelling or shrinkage that is caused by moisture variation is much higher than that caused by temperature variation. The driving potential of the thermal expansion can be attributed to the amplitude of the lattice vibration increasing when the temperature is increasing. For hygroexpansion, the water replaces the air to fill the holes between the solid matter of bamboo when the moisture content is increasing.
The difference in driving potentials of the mass transfer and energy transfer may lead to the different levels of deformation. The effect of moisture variation on the expansion of Moso bamboo-based building materials is much greater than the effect of temperature variation.

\subsection{Limitation and future directions}

This study examined the thermal expansion and hygroexpansion of Moso bamboo specimens by using one specimen for each direction. The results would be more representative if the number of specimens was to be increased in the future. Both thermal expansion and hygroexpansion experiments were only conducted for one cycle. Measurements for multiple cycles will be performed to characterise the periodic features of the expansion in future work. 


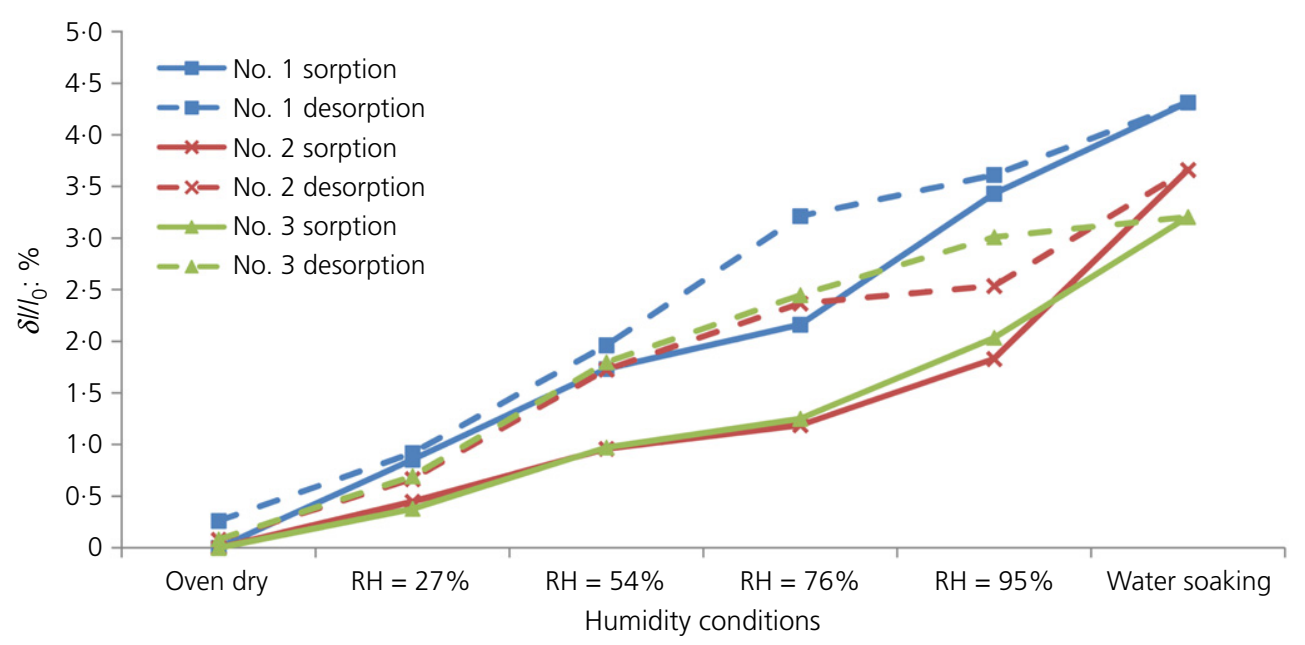

Figure 12. Hygroexpansion of node specimens in the tangential direction

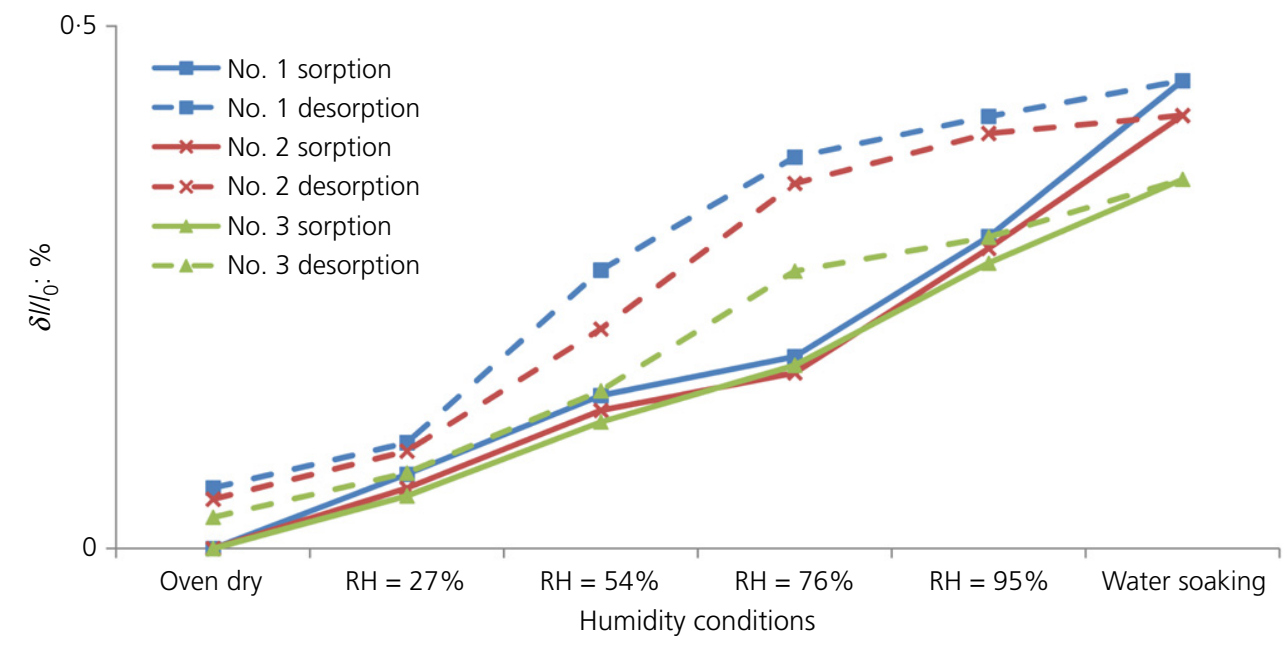

Figure 13. Hygroexpansion of internode specimens in the longitudinal direction

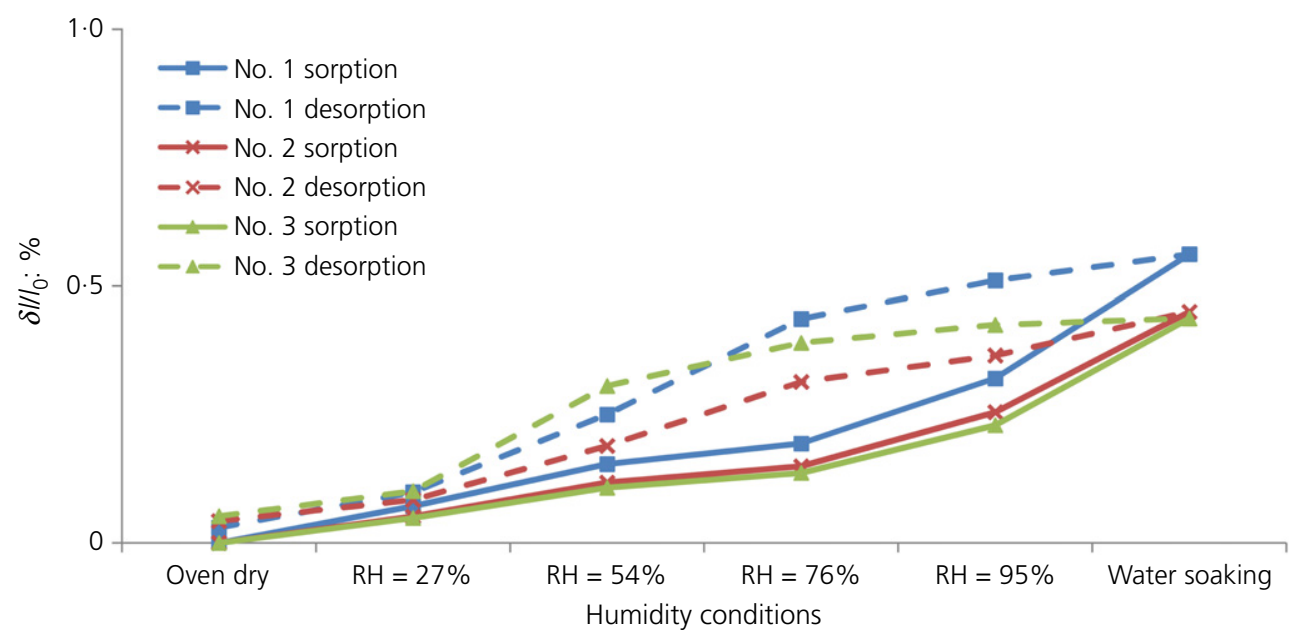

Figure 14. Hygroexpansion of node specimens in the longitudinal direction 


\section{Conclusion}

The thermal expansion and hygroexpansion of Moso bamboo specimens have been measured while increasing and subsequently decreasing temperature and moisture, respectively, thereby contributing to the scarce data in the literature. The thermal expansion measurements were conducted with a dilatometer. The hygroexpansion of bamboo specimens was measured with a micrometer and desiccators using different saturated solutions.

The thermal expansion of the bamboo specimens in the radial and the tangential directions is one order of magnitude higher than the thermal expansion in the longitudinal direction. The hygroexpansion results illustrated that the swelling of bamboo specimens increases with the RH, while the shrinkage of bamboo specimens decreases with reduction in RH. In the radial direction, the data indicated that the hygroexpansion values from high to low were for the external specimen, the middle specimen and the internal specimen, respectively. The hygroexpansion in the longitudinal direction is one order of magnitude lower than the hygroexpansion in the radial and tangential directions. The thermal expansion and hygroexpansion results showed that the swelling or shrinkage caused by moisture variation is much higher than that caused by temperature variation.

\section{REFERENCES}

Barber N and Meylan B (1964) The anisotropic shrinkage of wood. A theoretical model. Holzforschung 18(5): 146-156.

Chomcharn A and Skaar C (1983) Dynamic sorption and hygroexpansion of wood wafers exposed to sinusoidally varying humidity. Journal of the International Academy of Wood Science 17(4): 259-277.

Cverna F (2002) ASM Ready Reference: Thermal Properties of Metals ASM International, Russell Township, OH, USA.

Dong Q and Xiong Y (2014) Kinetics study on conventional and microwave pyrolysis of Moso bamboo. Bioresource Technology 171: 127-131, https://doi.org/10.1016/j.biortech.2014.08.063.

Fei Y (1995) Thermal expansion. In Mineral Physics and Crystallography: A Handbook of Physical Constants (Ahrens TJ (ed.)). American Geophysical Union, Washington, DC, USA, vol. 2, pp. 29-44.

Flander KD and Rovers R (2009) One laminated bamboo-frame house per hectare per year. Construction and Building Materials 23(1): 210-218.

Hendershot OP (1924) Thermal expansion of wood. Science 60(1559): 456- 457.

Hori R and Wada M (2005) The thermal expansion of wood cellulose crystals. Cellulose 12(5): 479-484.

Huang R, Zhang Y, Xu X, Zhou D and Wu Q (2012) Effect of hybrid mineral and bamboo fillers on thermal expansion behavior of bamboo fiber and recycled polypropylene-polyethylene composites. BioResources 7(4): 4563-4574.

Jiang Z, Zou Y, Ruan XWJ and Liu Y (2000) A study on the ultrastructure of bamboo cell wall by X-ray. Scientia Silvae Sinicae 36(3): $122-125$.

Li NP, Long JB, Su L, Wang L and Zhong S (2012) Experimental and theoretical study on thermal and moisture characteristics of new-type bamboo structure wall. Journal of Central South University 19(3): 600-608.

Li S, Wu S and Shen X (2011) A prediction model of neural networks for Phyllostachys pubescens' thermal conductivity. Chinese Agricultural Science Bulletin 27(31): 53-57.
Majumdar A, Mukhopadhyay S and Yadav R (2010) Thermal properties of knitted fabrics made from cotton and regenerated bamboo cellulosic fibres. International Journal of Thermal Sciences 49(10): 2042-2048.

Ma EN, Zhao GJ and Cao JZ (2005) Hygroexpansion of wood during moisture adsorption and desorption processes. Forestry Studies in China 7(2): 43-46.

Pentoney RH (1953) Mechanisms affecting tangential vs radial shrinkage. Forest Products Journal 3(2): 27-32.

Pizzo B, Rizzo G, Lavisci P, Megna B and Berti S (2002) Comparison of thermal expansion of wood and epoxy adhesives. Holz als Roh-und Werkstoff 60(4): 285-290.

Rafsanjani A, Stiefel M, Jefimovs K et al. (2014) Hygroscopic swelling and shrinkage of latewood cell wall micropillars reveal ultrastructural anisotropy. Journal of the Royal Society Interface 11(95), 20140126.

Skaar C (1988) Wood-Water Relations. Springer Verlag, Berlin, Germany.

Stevens W (1960) The thermal expansion of wood. Wood 25(8): 328-329.

Sun Z, Tian Y and An S (2006) Status of study on bamboo timber drying technology. World Forestry Research 19(6): 41-44.

Tu D and Xu B (2008) Character of hygrothermal and expansion effects of bamboo. Journal of Anhui Agricultural University 35(2): 178-180.

Van Der Lugt P, Van Den Dobbelsteen AAJF and Janssen JJA (2006) An environmental, economic and practical assessment of bamboo as a building material for supporting structures. Construction and Building Materials 20(9): 648-656.

Weatherwax RC and Stamm AJ (1956) The Coefficients of Thermal Expansion of Wood and Wood Products. US Forest Products Laboratory, Madison WI, USA, Report No. 1487.

Wu S, Yu S, Han J and Wu Y (2004) Testing and analysis of the thermodynamics parameters of Phyllostachys edulis Carr. H. de Leh. Journal of Central South Forestry University 24(5): 70-75.

Yu Y, Wang G, Qin D and Zhang B (2007) Variation in microfibril angle of bamboo by X-ray diffraction. Journal of Northeast Forestry University 35(8): 28-29.

\section{How can you contribute?}

To discuss this paper, please email up to 500 words to the editor at journals@ice.org.uk. Your contribution will be forwarded to the author(s) for a reply and, if considered appropriate by the editorial board, it will be published as discussion in a future issue of the journal.

Proceedings journals rely entirely on contributions from the civil engineering profession (and allied disciplines). Information about how to submit your paper online is available at www.icevirtuallibrary.com/page/authors, where you will also find detailed author guidelines. 